\title{
Decreased sensory nerve excitation and bone pain associated with mouse Lewis lung cancer in TRPV1-deficient mice.
}

Hiroki Wakabayashi ${ }^{1,4}$, Satoshi Wakisaka ${ }^{2}$, Toru Hiraga ${ }^{1,5}$, Kenji Hata $^{1}$, Riko Nishimura ${ }^{1}$, Makoto Tominaga $^{3}$, Toshiyuki Yoneda ${ }^{1,6}$

${ }^{1}$ Department of Biochemistry, and ${ }^{2}$ Oral Anatomy and Developmental Biology, Osaka University Graduate School of Dentistry, 1-8 Yamadaoka, Suita, Osaka 565-0871, Japan

${ }^{3}$ Okazaki Institute of Integrative Bioscience, National Institutes of Natural Sciences, 5-1 Higashiyama Myodaijicho, Okazaki, Aichi 444-8787, Japan

${ }^{4}$ Department of Orthopaedic Surgery, Mie University School of Medicine, 2-174 Edobashi, Tsu, Mie 514-8507, Japan

${ }^{5}$ Department of Histology and Cell Biology, Matsumoto Dental University, 1780 Gobara-Hirooka, Shiojiri, Nagano 399-0781, Japan

${ }^{6}$ Division of Hematology and Oncology, Indiana University School of Medicine, 980 W Walnut St, Indianapolis, IN 46202, USA.

Corresponding Author: Toshiyuki Yoneda: tyoneda@dent.osaka-u.ac.jp

Key Words: Bone metastasis, calcitonin gene-related peptide, osteoclasts, V-ATPase, protons, dorsal root ganglion

Conflict of Interest: Authors declare that they have no conflict of interest.

Acknowledgement: This study was supported by Princess Takamatsu Cancer Research

This is the author's manuscript of the article published in final edited form as:

Wakabayashi, H., Wakisaka, S., Hiraga, T., Hata, K., Nishimura, R., Tominaga, M., \& Yoneda, T. (2017). Decreased sensory nerve excitation and bone pain associated with mouse Lewis lung cancer in TRPV1-deficient mice. Journal of Bone and Mineral Metabolism, 1-12. https://doi.org/10.1007/s00774-017-0842-7 
Foundation (08-24020) and Naito Memorial Foundation of Science Promotion (200-07) to TY and Japan Society for the Promotion of Science, Grants-in-Aid for Scientific Research from the Ministry of Education, Culture, Sports, Science and Technology of Japan (17014058, 23390422, 23659870, 26293394, 26670806) to TY. 


\section{ABSTRACT}

Bone pain is one of the most common and life-limiting complications of cancer metastasis to bone. Although the mechanism of bone pain still remains poorly understood, bone pain is evoked as a consequence of sensitization and excitation of sensory nerves (SNs) innervating bone by noxious stimuli produced in the microenvironment of bone metastases. We showed that bone is innervated by calcitonin gene-related protein (CGRP) ${ }^{+}$SNs extending from dorsal root ganglia (DRG), the cell body of SNs, in mice. Mice intratibially injected with Lewis lung cancer (LLC) cells showed progressive bone pain evaluated by mechanical allodynia and flinching with increased $\mathrm{CGRP}^{+} \mathrm{SNs}$ in bone and augmented SN excitation in DRG as indicated by elevated numbers of pERK- and pCREB-immunoreactive neurons. Immunohistochemical examination of LLC-injected bone revealed that the tumor microenvironment is acidic. Bafilomycin A1, a selective inhibitor of $\mathrm{H}^{+}$secretion from vacuolar proton pump, significantly alleviated bone pain, indicating that the acidic microenvironment contributes to bone pain. We then determined if the transient receptor potential vanilloid 1 (TRPV1), a major acid-sensing nociceptor predominantly expressed on SNs, plays a role in bone pain by intratibially injecting LLC cells in TRPV1-deficient mice. Bone pain and SN excitation in DRG and spinal dorsal horn were significantly decreased in TRPV1-/- mice compared with wild-type mice. Our results suggest that TRPV1 activation on SNs innervating bone by the acidic cancer microenvironment in bone contributes to SN activation and bone pain. Targeting acid-activated TRPV1 is a potential therapeutic approach for cancer-induced bone pain. 


\section{INTRODUCTION}

Cancers including breast, prostate and lung cancer show a strong predilection for spreading to bone to develop bone metastases (1-3). Cancer metastasis to bone is associated with debilitating and life-limiting skeletal complications such as pathological fracture and spinal cord compression (4-6). In addition, bone pain is also one of the most common and devastating complications of bone metastasis and more than $70 \%$ of advanced cancer patients with bone metastases suffer from bone pain (4,7-9). Bone pain is associated with reduced performance status, increased fatigue, anxiety and depression, and reduced health quality of life, leading to increased morbidity and mortality (7-9). Treatment of bone pain is thus an important goal in the management of patients with advanced cancer who develop bone metastases. However, our understandings of the mechanism(s) underlying cancer-induced bone pain, which is required to logically develop effective treatments, are limited and currently-available therapies are inadequate and ineffective or often cause uncomfortable adverse effects (10).

Bone pain is induced as a consequence of sensitization and excitation of sensory nerves (SNs) innervating bone by mechanical, chemical and/or thermal noxious stimuli that are produced under complex interactions among cancer cells, bone cells (osteoclasts, osteoblasts and osteocytes), bone marrow stromal cells and immune cells $(11,12)$. Although the precise mechanism by which these noxious stimuli excite SNs and evoke bone pain remains unclear, clinical observations that bone-targeting agents including bisphosphonates and denosumab significantly relieve bone pain in cancer patients with bone metastases (13) indicate a critical role of osteoclasts in the pathophysiology of cancer-induced bone pain. Osteoclasts, which play a central role in the development and progression of bone metastasis by establishing the vicious cycle with metastatic cancer cells $(1,14,15)$, destroy bone by releasing protons via the plasma membrane a3 isoform vacuolar proton pump (16), thereby creating acidic microenvironments in bone metastases. Further, invasive and metastatic cancer cells show increased expression of 
plasma membrane vacuolar proton pump $(17,18)$ through which protons are actively extruded to avoid intracellular acidification due to elevated aerobic glycolysis (Warburg effect) (19). We reported that highly metastatic B16-F10 melanoma cells express increased plasma membrane vacuolar proton pump (20). Thus, the extracellular microenvironment of bone metastases is acidic. It is noted that acid/proton is a potent inducer of pain (21). These results collectively suggest that the acidic cancer microenvironment in bone plays a role in the induction of bone pain.

Peripheral SNs express nociceptors to recognize and transduce local noxious stimuli into electrochemical signals (21). The nociceptors transmit these noxious signals to the central nervous system (CNS) and brain via the dorsal root ganglion (DRG), the cell body of primary afferent SN fibers innervating peripheral tissues such as bone (22). The specific and major acid-sensing nociceptor of SNs is the transient receptor potential channel-vanilloid subfamily member 1 (TRPV1) (23). TRPV1, a member of TRP ion channel superfamily, is a $\mathrm{Ca}^{2+}$ channel that is predominantly expressed on nociceptive SNs and activated by capsaicin, heat $\left(>43^{\circ} \mathrm{C}\right)$ and acid (<pH 6.0) (24). TRPV1-/- mice showed little pain behavior in response to capsaicin, heat and inflammation (25). Of interest, a recent study reported that TRPV1-/- mice live longer with increased metabolic health than do wild-type (WT) mice, suggesting that reduced pain responses due to the absence of TRPV1 may increase longevity (26).

In the present study, we found that intratibial injection of Lewis lung cancer (LLC) cells in mice induced bone pain with increased number of TRPV1 ${ }^{+} \mathrm{SNs}$ in bone and excitation in DRG. Inhibition of the development of acidic microenvironment by bafilomycin A1 decreased bone pain. Importantly, SN excitation and bone pain following intratibial injection of LLC cells are significantly decreased in TRPV1-/- mice compared to wild-type (WT) mice. 


\section{MATERIALS AND METHODS}

\section{Reagents}

RPMI1640, bovine serum albumin (BSA) and rabbit anti-CGRP antibody were purchased from Sigma (St Louis, MO). Fetal calf serum (FCS) was from Asahi Glass Techno Corp. (Tokyo, Japan). Fluoro-ruby (Dextran tetramethylrhodamine 10,000 MW), Alexa Fluor 488-conjugated goat anti-rabbit IgG antibody, and 3-(2,4-dinitroanillino)-3'-amino-N-methyldipropylamine (DAMP) were purchased from Molecular Probes (Eugene, OR). Rabbit anti-dinitrophenol antibody was purchased from Oxford Bio Lab (Oxford, UK). FITC-conjugated anti-guinea pig IgG, Cy3-conjugated anti-guinea pig IgG, Cy5-conjugated anti-rabbit IgG and biotinylated anti-guinea pig IgG antibodies were purchased from Jackson Immuno Research Laboratories Inc. (West Grove, PA). Guinea pig anti-CGRP antibody was from Eurodiagnostica (Malmö, Sweden). Normal swine serum (NSS) and biotinylated swine anti-rabbit IgG antibody were purchased from Dako, (Copenhagen, Denmark). ABC complex, 3,3'-diaminobenzidine (DAB) substrate kit and Vectorshield were purchased from Vector Laboratories, Inc.(Burlingame, CA). Rabbit anti-phosphorylated ERK antibody was purchased from Cell Signaling Technology, (Danvers, MA). Rabbit anti-c-fos and rabbit anti-TRPV1 antibodies were purchased from Oncogene Science (Cambridge, MA, USA). Bafilomycin A1 was purchased from Wako Pure Chemical Industries, Ltd. (Osaka, Japan). Carboxylate cement (dental resin) was purchased from Unifast Trad, GC Dental Products Co., (Aichi, Japan). Silk threads were from Ethicon (Somerville, NJ)

\section{Cell culture}

Mouse LLC cells were purchased from Cell Resource Center for Biomedical Research, Tohoku University (Miyagi, Japan) and cultured in RPMI640 medium supplemented with 10\% FCS, 100 $\mu \mathrm{g} / \mathrm{ml}$ kanamycin sulfate (Meiji Seika, Ltd., Tokyo, Japan) in a humidified atmosphere of $5 \% \mathrm{CO}_{2}$. An earlier study reported that intra-femoral injection of LLC cells in mice induced bone pain (27). 


\section{Animals}

C57BL/6 mice (4-6 week-old, 20-25 g) were purchased from Japan SLC Inc. (Hamamatsu, Japan). TRPV1-/- mice were kindly provided by Dr. Julius of the Department of Cellular and Molecular Pharmacology at UCSF. Mice were maintained on a 12:12 h dark-light cycle with food and water. All experiments were conducted in accordance with the ethical guidelines of the International Association for the Study of Pain (28) and approved by the Institutional Animal Care and Use Committee of Osaka University Graduate School of Dentistry.

\section{Intratibial injection of LLC cells}

All procedures were performed under general anesthesia with pentobarbital $(0.05 \mathrm{mg} / \mathrm{g}$ body weight; Dainippon Pharmaceutical Co., Ltd., Osaka, Japan).

Surgical procedures were performed as described previously (29). Following anesthesia, mice were placed abdominal side up. A one cm-long rostro-caudal incision was made in the skin over the top half of the right tibiae. The tibiae were carefully exposed with minimal damages to the muscle or blood vessels. Using a 30-gauge needle, bone was pierced $2 \mathrm{~mm}$ below the knee joint distal to the epiphyseal growth plate and needle was inserted into the marrow cavity of tibiae. The 30-gauge needle was then removed and replaced with a 29-gauge needle, and LLC cells (1 $x 10^{5}$ ) in $10 \mu \mathrm{l}$ PBS were slowly injected through a $25 \mu$ l Hamilton syringe needle that was inserted in the inner hole of the 29-gauge needle to prevent leakage of cells. Sham mice received PBS without cells. The injection hole on bone was closed using carboxylate cement and skin wound using silk threads. In some experiments, mice were treated with bafilomycin A1 (25 $\mu \mathrm{g} / \mathrm{kg} /$ day) subcutaneous injection once a day from day 7 to day 14 . Control mice received PBS. Tumor growth in bone was monitored weekly by X-ray. Mice in which LLC cells in the bone marrow broke out the cortex and spread on the periosteum were got rid of from the experiments. 


\section{Assessment for bone pain}

Bone pain was determined by widely-used pain-related behavior tests including stimulus-dependent thermal plantar hyperalgesia test and stimulus-independent spontaneous hind-limb lifting test (30). A few days prior to intratibial LLC cell inoculation, mice were subjected to these tests to obtain the data of control behavior. Tests were repeated three times by a single investigator in a blind manner. Mice in which cancer cells broke out the cortex and spread on periosteum were not included in the tests, so that the bone pain observed here represents bone pain that is primarily induced by LLC cells within bone marrow.

\section{$\underline{\text { Thermal plantar hyperalgesia test }}$}

Plantar test (Plantar test, 7370; Ugo Basile, Comerio, Italy) was performed as described previously (31). Mice were placed in a clear plastic chamber with a glass floor unrestrained and allowed to acclimate to their environment for $30 \mathrm{~min}$ before testing. Radiant heat source was then positioned under the glass floor directly beneath the hind-paw. A photoelectric cell aimed at the aperture-detected light reflects from the paw and turned off the electronic clock when paw movement interrupted the reflected light. Time required for mice to withdraw their paw of LLC-injected tibiae in response to radiant heat stimulation was determined using an electronic clock circuit connected with a microcomputer.

\section{Hind-limb lifting (Flinching) test}

Mice were placed on a glass floor at room temperature (32). After an acclimation for 30 min, mice were determined for cumulative duration or frequency of repetitive and spontaneous flinching of the LLC-injected hind-limbs (rapid lifting of the entire hind-limb that begins with hip flexion and includes dorsflexion of the toes) for $10 \mathrm{~min}$. 


\section{Retrograde tracing}

Retrograde tracing was performed to verify that SNs in lumbar DRG (L3-L5) innervate tibial bone marrow cavity according to the methods described with modifications (33). Mice were anesthetized and the retrograde neural tracer, 5\% fluoro-ruby (Dextran tetramethylrhodamine $10,000 \mathrm{MW})(4 \mu \mathrm{l})$, was injected into the medullary cavity of tibiae. The tracer injection site was covered with carboxylate cement. Two days after the tracer injection, animals were anesthetized with pentobarbital and perfused through left cardiac ventricle with $0.1 \mathrm{M}$ PBS followed by $4 \%$ paraformaldehyde fixative as we reported (34). DRGs (L3-5) were subsequently harvested and processed for the preparation of cryostat sections and double-stained using a rabbit anti-TRPV1 antibody (1:500) and guinea pig anti-CGRP antibody (1:5000). Secondary antibodies conjugated with Cy5 and FITC (1:500) were used to distinguish the immuno-labeling from the labeling with fluoro-ruby. Stained sections were examined under a confocal laser scanning microscope (LSM510, Ver 3.2, Carl Zeiss).

\section{Immunohistochemistry (IHC)}

\section{Dorsal root ganglion (DRG) and spinal cord}

At the end of experiments, mice were anesthetized, perfused and DRG (L3-L5) and spinal cord (lamina II/III) were removed, post-fixed overnight in the same fixative and then cryo-protected in $20 \%$ sucrose overnight at $4^{\circ} \mathrm{C}$. Sections $(10-30 \mu \mathrm{m})$ were cut on a cryostat. Following rinsing with PBS, sections were treated with PBS containing $0.3 \% \mathrm{H}_{2} \mathrm{O}_{2}$ to block endogenous peroxidase activity for $30 \mathrm{~min}$ at room temperature (RT), rinsed with PBS several times, treated with PBS containing $1 \%$ normal swine serum and 1\% BSA for $30 \mathrm{~min}$, and then incubated with a rabbit anti-TRPV1 (1:500), rabbit anti-CGRP (1:10,000), phosphorylated ERK $(1: 1,000)$ or c-Fos $(1: 2,000)$ overnight at RT. These sections were then incubated with biotinylated swine anti-rabbit

$\lg G$ (1:500) and subsequently with the $A B C$ complex at RT for 90 min each. The 
antigen-antibody reaction sites were visualized by incubation with $0.04 \%$ 3,3'-diaminobenzidine (DAB) and $0.003 \% \mathrm{H}_{2} \mathrm{O}_{2}$ in $0.05 \mathrm{M}$ Tris- $\mathrm{HCl}$ buffer $(\mathrm{pH} 7.5)$ intensified with $0.1 \%$ nickel ammonium sulfate. The primary and secondary antibodies were diluted with PBS containing $1 \%$ NSS and $1 \%$ BSA. The immunostained sections were mounted onto gelatin-subbed glass slides, dehydrated through an ascending series of ethanol, cleared, and cover-slipped with Permount and enumerated for numbers of pERK- and c-fos-immunoreactive SNs under a light microscope.

\section{$\underline{\text { Bone }}$}

After fixation with $10 \%$ neutral formalin, bones were harvested and decalcified in $10 \%$ EDTA for 2 weeks. Sections $(30 \mu \mathrm{m})$ were cut on a cryostat, rinsed with PBS several times, treated with PBS containing $1 \%$ NSS and $1 \%$ BSA for $30 \mathrm{~min}$, and then incubated with a rabbit anti-TRPV1 (1:100) and guinea pig anti-CGRP $(1: 2,000)$ overnight at RT. These sections were then incubated with Alexa Fluor 488-conjugated goat anti-rabbit IgG (1:500) and Cy3-conjugated goat anti-guinea pig IgG (1:500) at RT for 90 min. The immunostained sections were cover-slipped with Vectorshield. Sections were examined for $\mathrm{CGRP}^{+}$and TRPV1 ${ }^{+}$SNs under a confocal laser scanning microscope. The images were captured with CCD camera software (AxioCom Ver. 4.6; Carl Zeiss), and transferred to Adobe Photoshop.

Histochemical staining for tartrate-resistant acid phosphatase (TRAP) was also performed and numbers of TRAP ${ }^{+}$osteoclasts were enumerated as we described previously (35).

\section{Detection of acidic microenvironment}

At day 14, 300 mg 3-(2,4-dinitroanillino)-3'-amino-N-methyldipropylamine (DAMP), which selectively accumulates in acidic microenvironments, in PBS $(1.2 \mathrm{mg} / \mathrm{ml})$ was administered via the carotid artery over 30 min to cancer-injected mice as described previously (36). After fixation with $4 \%$ paraformaldehyde through left cardiac ventricle, bones were decalcified in $10 \%$ EDTA 
for 2 weeks. Paraffin sections were made, incubated with rabbit anti-dinitrophenol antibody (1:50, Oxford Bio Lab), which cross-reacts with DAMP, overnight at RT and then with the ABC complex. The antigen-antibody reaction sites were visualized by incubation with $0.04 \%$ 3,3'-diaminobenzidine (DAB) and $0.003 \% \mathrm{H}_{2} \mathrm{O}_{2}$ in $0.05 \mathrm{M}$ Tris- $\mathrm{HCl}$ buffer $(\mathrm{pH} 7.5)$ intensified with $0.08-0.1 \%$ nickel ammonium sulfate. Sections were counterstained with methyl green

\section{Statistical analysis}

The data were analyzed by one-way ANOVA followed by Fisher's PLSD post-hoc test (StatView; SAS Institute Inc., Cary, NC). P values of $<0.05$ were considered statistically significant. All of the data were presented as mean \pm SE. All experiments were performed at least three times with identical results. 


\section{RESULTS}

\section{Innervation of SNs in bone}

Data are accumulating that bone is densely innervated by SNs $(33,37-40)$. Consistent with these earlier studies, our IHC examination using an antibody for calcitonin gene-related peptide (CGRP), which is a widely-used marker for SN (41), demonstrated that CGRP-positive (CGRP $\left.{ }^{+}\right)$ SNs accumulate at periosteum, extending into the medullary cavity through mineralized bone (Figure 1A, arrows). Further, $\mathrm{CGRP}^{+} \mathrm{SNs}$ (Figure 1B, arrows) locate in the close proximity of TRAP $^{+}$osteoclasts (Figure 1B, arrow head) in bone. These results suggest that SNs can be excited by osteoclasts during bone destruction and are consistent with the clinical observations that the specific inhibitors of osteoclasts, bisphosphonates and denosumab, relieve bone pain in cancer patients with bone metastases (13). Of note, $\mathrm{CGRP}^{+} \mathrm{SNs}$ in bone co-expressed TRPV1 (Figure 1C), suggesting that $\mathrm{CGRP}^{+} \mathrm{SNs}$ are excited to evoke bone pain by acidic bone microenvironments via TRPV1. Retrograde tracing using fluoro-ruby reveals that the bone marrow cavity of tibiae is innervated by fluoro-ruby ${ }^{+}, \mathrm{CGRP}^{+}$and TRPV1 ${ }^{+} \mathrm{SNs}$, demonstrating that these SNs extend from L3-L5 DRG (Figure 1D).

\section{Animal model of cancer-induced bone pain}

LLC cells inoculated into the medullary cavity of right tibiae developed osteolytic lesions on X-ray (Figure 2A, right, arrow) and occupied intramedullary cavity (Figure 2A, left, asterisks) over 14 days. In parallel with intramedullary growth of LLC cells, numbers of TRAP ${ }^{+}$osteoclasts in tibiae were increased (Figure 2B). Importantly, we found that numbers of $\mathrm{CGRP}^{+}$and $\mathrm{TRPV}^{+} \mathrm{SNs}$ were also increased in tibiae injected with LLC cells (Figure 2C). Other investigators also reported that sprouting of SNs is increased in the presence of tumor in bone $(39,42)$. More importantly, mice intratibially injected with LLC cells began to demonstrate thermal hyperalgesia at day 7 compared with sham mice (Figure 2D) that continuously progressed until day 14. Further, 
these mice also exhibited increased frequency (Figure 2E) and duration (Figure 2F) of lifting of their hind-limbs (flinching) injected with LLC cells compared with sham mice. Sham mice (Figure 2D, 2E \& 2F) exhibited no thermal hyperalgesia and lifting of their hind-limbs. Left tibiae of LLC-injected mice had no evidence of bone pain (data not shown), demonstrating that bone pain is associated with local LLC colonization rather than a systemic effect of LLC cells. These results show that LLC cells intratibially injected in mice promote SN sprouting and progressively induce bone pain.

\section{Role of acidic bone microenvironment in cancer-induced bone pain}

To determine if LLC cells growing in bone develop acidic microenvironment in bone, we administered DAMP, which selectively accumulates in acidic compartments and is immunohistochemically detected by anti-dinitrophenol antibody, which cross-reacts with DAMP (36), to LLC-injected mice. As shown in Figure 3A, the microenvironments where LLC cells are progressively growing showed DAMP accumulation and thus are acidic. Further, consistent with our previous report (43), the microenvironments associated with $\mathrm{TRAP}^{+}$osteoclasts were also acidic (Figure 3B). The $\mathrm{pH}$ value of the microenvironment associated with LLC cells and osteoclasts was unknown.

To determine the role of the acidic microenvironment developed in LLC-growing bone in the induction of bone pain, we tested the selective inhibitor of vacuolar proton pump, bafilomycin A1, which, we reported, reduces inflammatory bone pain (44), in LLC-injected mice. We found that bafilomycin A1 significantly decreased thermal hyperalgesia (Figure 3C) and flinching (Figure 3D). These results suggest that bone pain is induced by the acidic microenvironment created as a result of LLC tumor progression in bone. It should be, however, noted that bafilomycin A1 only partially decreased thermal hyperalgesia, suggesting that mechanisms other than acidic cancer microenvironment also contribute to the bone pain in this model. 


\section{Role of TRPV1 in cancer-induced bone pain}

We then determined if the acid-sensing nociceptor TRPV1 is involved in the induction of bone pain in mice carrying LLC cancer in bone. To approach this, we intratibially injected LLC cells in TRPV1-/- mice and WT mice and conducted pain behavior tests in these mice. LLC tumor growth in tibiae in TRPV1-/- mice was not different from that in WT mice during 14 day experimental period (data not shown). Intratibial injection of LLC cells induced thermal hyperalgesia (Figure 4A) and flinching (Figure 4C and 4D) in WT mice. Importantly, however, TRPV1-/- mice showed no thermal hyperalgesia (Figure 4B) and significantly decreased flinching (Figure 4C and 4D). These results suggest that TRPV1 on SNs plays a critical role in LLC cancer-induced bone pain.

\section{Role of TRPV1 in SN excitation}

Since cancer-induced bone pain was decreased in TRPV1-/- mice, we next determined if SN excitation is also reduced in TRPV1-/- mice by assessing the expression of phosphorylated extracellular signal-regulated protein kinase (pERK) in DRG (the primary afferent neurons) and c-fos in the spinal dorsal horn (the secondary afferent neurons). pERK is a widely used molecular indicator for neural activation by noxious stimuli (45) and c-fos is a marker for efficient detection of SNs excited in response to nociceptive stimuli (46).

IHC showed that the number of $p E R K^{+}$neurons in DRG was significantly increased following intratibial injection of LLC cells into WT mice (Figure 5A, 5B and 5E). In contrast, intratibial injection of LLC cells did not increase the number of $p E R K^{+}$neurons in DRG in TRPV1-/- mice (Figure 5C, 5D and 5E).

Similarly, WT mice showed significant increase in c-fos ${ }^{+}$neurons in spinal dorsal horn following intratibial injection of LLC cells (Figure 6A, 6B and 6E), whereas no change in the number c-fos ${ }^{+}$ neurons in spinal dorsal horn was seen in TRPV1-/- mice intratibially injected with LLC cells (Figure 6C, 6D and 6E).

\section{DISCUSSION}


Mechanism of cancer-induced bone pain is poorly understood and bone pain is undertreated in cancer patients with bone metastases (7-9). Since progression of bone metastases is modulated by the interactive co-operations (vicious cycle) between bone-colonizing cancer cells and bone-destroying osteoclasts with supports of other bone cells including osteoblasts and potentially osteocytes $(1,14,15)$, it is likely that these players also contribute to the pathophysiology of bone pain associated with bone metastases. In the present study, we found that cancer cells colonizing bone and bone-resorbing osteoclasts play a critical role in the induction of bone pain through creation of an acidic tumor microenvironment. Our results demonstrated that treatment of LLC tumor-bearing mice with bafilomycin A1, which, we recently reported, blocks the development of acidic microenvironment in cancer-colonized bone through inhibiting proton release via the plasma membrane vacuolar proton pump (43), significantly reduced bone pain. These results suggest that bone pain associated with bone metastasis is attributable to the acidic tumor microenvironment created by increased proton release by cancer cells and bone-destroying osteoclasts.

These results led us to determine if the acid-sensing nociceptor TRPV1 expressed on SNs is involved in the pathophysiology of bone pain associated with the acidic tumor microenvironment by a genetic approach using TRPV1-/- mice. Several earlier studies reported that inhibition of TRPV1 activation using selective TRPV1 antagonists reduced bone pain in preclinical animal models (47-50), demonstrating a critical role of TRPV1 in bone pain. However, one concern in these pharmacological studies is that the results obtained could be due to off-target effects of antagonists tested. In this study, we found that SN excitation as determined by the number of pERK ${ }^{+}$SNs in DRG and c-fos ${ }^{+}$SNs in spinal dorsal horn was significantly reduced and bone pain was also significantly decreased in TRPV1-/- mice intratibially injected with LLC cells compared with WT mice. Our genetic approach using TRPV1-I- mice thus provide the concrete evidence that TRPV1 contributes to SN excitation and bone pain induced in response to the acidic tumor 
microenvironment in bone.

It has been long proposed that the periosteum is the primary site from which bone pain elicits (7). However, cancers growing within bone marrow or mineralized bone often induce bone pain in the absence of evident periosteal involvement (38), indicating the innervation of $\mathrm{SN}$ fibers in non-periosteal sites in bone. Consistent with this notion, our IHC showed that $\mathrm{CGRP}^{+} \mathrm{SN}$ fibers not only accumulate at the periosteum but also terminate into the bone marrow cavity running through mineralized bone. Importantly, we found that cancer colonization increased sprouting of these bone-innervating SN fibers and up-regulated excitation of these SN fibers at DRG (L3-L5) and spinal dorsal horn via TRPV1 activation in response to the acidic microenvironment of cancer-colonized bone. Consequence of these changes in SNs is obviously parts of the mechanism underlying cancer-induced bone pain. Thus, bone is an organ in which SNs heavily innervate and show noxious responses to changes in the microenvironment resulting from cancer colonization and osteoclastic bone destruction and provides a unique model to study the pathophysiology of cancer-induced pain.

Bone fractures are one of the major causes of bone pain (7) and the bone pain observed in this study could result from pathologic bone microfractures associated with increased osteolysis. Unfortunately, however, the behavior tests and biochemical determinations used in this study do not allow us to distinguish bone pain due to bone microfractures from that caused by acidic tumor microenvironment. Our results show that mice intratibially injected with LLC cells began to manifest nocifensive behaviors at day 7 at which bone destruction by LLC cells was minimum on X-ray and evident bone microfractures were not verified by histology. These results suggest that contribution of bone $\underline{\text { microfractures to bone pain is minimal in early stages of the disease in this model. However, at day } 14}$ at which bone destruction overtly advanced, bone fractures most likely caused to increase bone pain. We think that contribution of bone fractures to cancer-induced bone pain is stage-dependent at least in this model. Intratibial injection of cancer cells may mechanically disrupt the normal innervation of bone 
marrow. In fact, it should be noted that sham mice intratibially injected with PBS showed thermal hyperalgesia at day 1 and 4, suggesting that traumatic injuries by needle insertion into the medullary cavity caused thermal hyperalgesia. However, the thermal hyperalgesia was no longer seen at day 7 and thereafter, indicating that the injuries were healed by day 7 . Thus, thermal hyperalgesia and flinching observed in LCC-injected mice after day 7 are ascribed to tumor progression, representing cancer-induced bone pain.

In conclusion, our results show that TRPV1 activation by the acidic cancer microenvironment in bone promotes SN sprouting and excitation, leading to bone pain. Blocking TRPV1 activation by acidic cancer microenvironment may be a novel and potential therapeutic approach for cancer-induced bone pain. 


\section{REFERENCES}

1. Weilbaecher, K. N., Guise, T. A., and McCauley, L. K. (2011) Cancer to bone: a fatal attraction. Nat Rev Cancer 11, 411-425

2. Randall, R. L. (2014) A promise to our patients with metastatic bone disease. Ann Surg Oncol 21, 4049-4050

3. Coleman, R. E., Gregory, W., Marshall, H., Wilson, C., and Holen, I. (2013) The metastatic microenvironment of breast cancer: clinical implications. Breast 22 Suppl 2 , S50-56

4. Coleman, R. E. (2006) Clinical features of metastatic bone disease and risk of skeletal morbidity. Clin Cancer Res 12, 6243s-6249s

5. Saad, F., Ivanescu, C., Phung, D., Loriot, Y., Abhyankar, S., Beer, T. M., Tombal, B., and Holmstrom, S. (2017) Skeletal-related events significantly impact health-related quality of life in metastatic castration-resistant prostate cancer: data from PREVAIL and AFFIRM trials. Prostate Cancer Prostatic Dis

6. von Moos, R., Costa, L., Ripamonti, C. I., Niepel, D., and Santini, D. (2017) Improving quality of life in patients with advanced cancer: Targeting metastatic bone pain. Eur $\mathrm{J}$ Cancer 71, 80-94

7. Mercadante, S. (1997) Malignant bone pain: pathophysiology and treatment. Pain 69, $1-18$

8. Mantyh, P. (2013) Bone cancer pain: causes, consequences, and therapeutic opportunities. Pain 154 Suppl 1, S54-62

9. Falk, S., and Dickenson, A. H. (2014) Pain and nociception: mechanisms of cancer-induced bone pain. J Clin Oncol 32, 1647-1654

10. Dalal, S., and Bruera, E. (2013) Access to opioid analgesics and pain relief for patients with cancer. Nat Rev Clin Oncol 10, 108-116 
11. Yoneda, T., Hiasa, M., Nagata, Y., Okui, T., and White, F. A. (2015) Acidic microenvironment and bone pain in cancer-colonized bone. Bonekey Rep 4, 690

12. Yoneda, T., Hiasa, M., Nagata, Y., Okui, T., and White, F. (2015) Contribution of acidic extracellular microenvironment of cancer-colonized bone to bone pain. Biochim Biophys Acta 1848, 2677-2684

13. Patrick, D. L., Cleeland, C. S., von Moos, R., Fallowfield, L., Wei, R., Ohrling, K., and Qian, Y. (2015) Pain outcomes in patients with bone metastases from advanced cancer: assessment and management with bone-targeting agents. Support Care Cancer 23, $1157-1168$

14. Mundy, G. R. (2002) Metastasis to bone: causes, consequences and therapeutic opportunities. Nat Rev Cancer 2, 584-593

15. Yoneda, T., Tanaka, S., and Hata, K. (2013) Role of RANKL/RANK in primary and secondary breast cancer. World J Orthop 4, 178-185

16. Qin, A., Cheng, T. S., Pavlos, N. J., Lin, Z., Dai, K. R., and Zheng, M. H. (2012) V-ATPases in osteoclasts: structure, function and potential inhibitors of bone resorption. Int J Biochem Cell Biol 44, 1422-1435

17. Gatenby, R. A., Gawlinski, E. T., Gmitro, A. F., Kaylor, B., and Gillies, R. J. (2006) Acid-mediated tumor invasion: a multidisciplinary study. Cancer Res 66, 5216-5223

18. Cotter, K., Capecci, J., Sennoune, S., Huss, M., Maier, M., Martinez-Zaguilan, R., and Forgac, M. (2015) Activity of plasma membrane V-ATPases is critical for the invasion of MDA-MB231 breast cancer cells. J Biol Chem 290, 3680-3692

19. Parks, S. K., Chiche, J., and Pouyssegur, J. (2013) Disrupting proton dynamics and energy metabolism for cancer therapy. Nat Rev Cancer 13, 611-623

20. Nishisho, T., Hata, K., Nakanishi, M., Morita, Y., Sun-Wada, G. H., Wada, Y., Yasui, N., and Yoneda, T. (2011) The a3 isoform vacuolar type $\mathrm{H}(+)$-ATPase promotes distant metastasis in the mouse B16 melanoma cells. Mol Cancer Res 9, 845-855 
21. Basbaum, A. I., Bautista, D. M., Scherrer, G., and Julius, D. (2009) Cellular and molecular mechanisms of pain. Cell 139, 267-284

22. Krames, E. S. (2015) The dorsal root ganglion in chronic pain and as a target for neuromodulation: a review. Neuromodulation 18, 24-32; discussion 32

23. Lozano-Ondoua, A. N., Symons-Liguori, A. M., and Vanderah, T. W. (2013) Cancer-induced bone pain: Mechanisms and models. Neurosci Lett 557 Pt A, 52-59

24. Julius, D. (2013) TRP channels and pain. Annu Rev Cell Dev Biol 29, 355-384

25. Caterina, M. J., Leffler, A., Malmberg, A. B., Martin, W. J., Trafton, J., Petersen-Zeitz, K. R., Koltzenburg, M., Basbaum, A. I., and Julius, D. (2000) Impaired nociception and pain sensation in mice lacking the capsaicin receptor. Science $\mathbf{2 8 8 , 3 0 6 - 3 1 3}$

26. Riera, C. E., Huising, M. O., Follett, P., Leblanc, M., Halloran, J., Van Andel, R., de Magalhaes Filho, C. D., Merkwirth, C., and Dillin, A. (2014) TRPV1 pain receptors regulate longevity and metabolism by neuropeptide signaling. Cell 157, 1023-1036

27. Isono, M., Suzuki, T., Hosono, K., Hayashi, I., Sakagami, H., Uematsu, S., Akira, S., DeClerck, Y. A., Okamoto, H., and Majima, M. (2011) Microsomal prostaglandin E synthase-1 enhances bone cancer growth and bone cancer-related pain behaviors in mice. Life Sci 88, 693-700

28. Zimmermann, M. (1983) Ethical guidelines for investigations of experimental pain in conscious animals. Pain 16, 109-110

29. Medhurst, S. J., Walker, K., Bowes, M., Kidd, B. L., Glatt, M., Muller, M., Hattenberger, M., Vaxelaire, J., O'Reilly, T., Wotherspoon, G., Winter, J., Green, J., and Urban, L. (2002) A rat model of bone cancer pain. Pain 96, 129-140

30. Slosky, L. M., Largent-Milnes, T. M., and Vanderah, T. W. (2015) Use of Animal Models in Understanding Cancer-induced Bone Pain. Cancer Growth Metastasis 8, 47-62

31. Hargreaves, K., Dubner, R., Brown, F., Flores, C., and Joris, J. (1988) A new and sensitive method for measuring thermal nociception in cutaneous hyperalgesia. Pain $\mathbf{3 2}$, 
77-88

32. Shimoyama, M., Tanaka, K., Hasue, F., and Shimoyama, N. (2002) A mouse model of neuropathic cancer pain. Pain 99, 167-174

33. Ivanusic, J. J. (2009) Size, neurochemistry, and segmental distribution of sensory neurons innervating the rat tibia. J Comp Neurol 517, 276-283

34. Nakanishi, M., Hata, K., Nagayama, T., Sakurai, T., Nishisho, T., Wakabayashi, H., Hiraga, T., Ebisu, S., and Yoneda, T. (2010) Acid activation of Trpv1 leads to an up-regulation of calcitonin gene-related peptide expression in dorsal root ganglion neurons via the CaMK-CREB cascade: a potential mechanism of inflammatory pain. Mol Biol Cell 21, 2568-2577

35. Hiraga, T., Myoui, A., Hashimoto, N., Sasaki, A., Hata, K., Morita, Y., Yoshikawa, H., Rosen, C. J., Mundy, G. R., and Yoneda, T. (2012) Bone-derived IGF mediates crosstalk between bone and breast cancer cells in bony metastases. Cancer Res 72, 4238-4249

36. Verlander, J. W., Madsen, K. M., Larsson, L., Cannon, J. K., and Tisher, C. C. (1989) Immunocytochemical localization of intracellular acidic compartments: rat proximal nephron. Am J Physiol 257, F454-462

37. Serre, C. M., Farlay, D., Delmas, P. D., and Chenu, C. (1999) Evidence for a dense and intimate innervation of the bone tissue, including glutamate-containing fibers. Bone $\mathbf{2 5}$, 623-629

38. Mach, D. B., Rogers, S. D., Sabino, M. C., Luger, N. M., Schwei, M. J., Pomonis, J. D., Keyser, C. P., Clohisy, D. R., Adams, D. J., O'Leary, P., and Mantyh, P. W. (2002) Origins of skeletal pain: sensory and sympathetic innervation of the mouse femur. Neuroscience $113,155-166$

39. Jimenez-Andrade, J. M., Mantyh, W. G., Bloom, A. P., Xu, H., Ferng, A. S., Dussor, G., Vanderah, T. W., and Mantyh, P. W. (2010) A phenotypically restricted set of primary afferent nerve fibers innervate the bone versus skin: therapeutic opportunity for treating 
skeletal pain. Bone 46, 306-313

40. Fukuda, T., Takeda, S., Xu, R., Ochi, H., Sunamura, S., Sato, T., Shibata, S., Yoshida, Y., Gu, Z., Kimura, A., Ma, C., Xu, C., Bando, W., Fujita, K., Shinomiya, K., Hirai, T., Asou, Y., Enomoto, M., Okano, H., Okawa, A., and Itoh, H. (2013) Sema3A regulates bone-mass accrual through sensory innervations. Nature 497, 490-493

41. Benemei, S., Nicoletti, P., Capone, J. G., and Geppetti, P. (2009) CGRP receptors in the control of pain and inflammation. Curr Opin Pharmacol 9, 9-14

42. Mantyh, P. W. (2014) The neurobiology of skeletal pain. Eur J Neurosci 39, 508-519

43. Hiasa, M., Okui, T., Allette, Y. M., Ripsch, M. S., Sun-Wada, G. H., Wakabayashi, H., Roodman, G. D., White, F. A., and Yoneda, T. (2017) Bone Pain Induced by Multiple Myeloma Is Reduced by Targeting V-ATPase and ASIC3. Cancer Res

44. Nagae, M., Hiraga, T., Wakabayashi, H., Wang, L., Iwata, K., and Yoneda, T. (2006) Osteoclasts play a part in pain due to the inflammation adjacent to bone. Bone 39 , $1107-1115$

45. Doya, H., Ohtori, S., Takahashi, K., Aoki, Y., Ino, H., Takahashi, Y., Moriya, H., and Yamashita, T. (2005) Extracellular signal-regulated kinase mitogen-activated protein kinase activation in the dorsal root ganglion (DRG) and spinal cord after DRG injury in rats. Spine (Phila Pa 1976) 30, 2252-2256

46. Coggeshall, R. E. (2005) Fos, nociception and the dorsal horn. Prog Neurobiol 77, 299-352

47. Ghilardi, J. R., Rohrich, H., Lindsay, T. H., Sevcik, M. A., Schwei, M. J., Kubota, K., Halvorson, K. G., Poblete, J., Chaplan, S. R., Dubin, A. E., Carruthers, N. I., Swanson, D., Kuskowski, M., Flores, C. M., Julius, D., and Mantyh, P. W. (2005) Selective blockade of the capsaicin receptor TRPV1 attenuates bone cancer pain. J Neurosci 25, 3126-3131

48. Niiyama, Y., Kawamata, T., Yamamoto, J., Omote, K., and Namiki, A. (2007) Bone cancer increases transient receptor potential vanilloid subfamily 1 expression within distinct 
subpopulations of dorsal root ganglion neurons. Neuroscience 148, 560-572

49. Niiyama, Y., Kawamata, T., Yamamoto, J., Furuse, S., and Namiki, A. (2009) SB366791, a TRPV1 antagonist, potentiates analgesic effects of systemic morphine in a murine model of bone cancer pain. Br J Anaesth 102, 251-258

50. Fuseya, S., Yamamoto, K., Minemura, H., Yamaori, S., Kawamata, T., and Kawamata, M. (2016) Systemic QX-314 Reduces Bone Cancer Pain through Selective Inhibition of Transient Receptor Potential Vanilloid Subfamily 1-expressing Primary Afferents in Mice. Anesthesiology 125, 204-218 


\section{FIGURE LEGEND}

\section{Figure 1}

A. Innervation of $\mathrm{CGRP}^{+} \mathrm{SN}$ in bone. Sections $(30 \mu \mathrm{m}$ thickness) of mouse bone were immunostained with anti-CGRP antibody, counterstained with methyl green and examined under a light microscope (x 100). Arrows: CGRP ${ }^{+}$SNs

B. Spatial relationship between SNs and osteoclasts in bone. Sections were immunostained with anti-CGRP antibody and subsequently stained for TRAP (x 400). Arrows: CGRP ${ }^{+}$ SNs; arrowhead: TRAP $^{+}$osteoclast.

C. Innervation of $\mathrm{CGRP}^{+}$and $\mathrm{TRPV}^{+} \mathrm{SN}$ in bone. Sections were immunostained with anti-CGRP and anti-TRPV1 antibody and examined under a confocal laser scanning microscope (x 200). Arrows: CGRP ${ }^{+}$and $\mathrm{TRPV}^{+} \mathrm{SN}$.

D. Retrograde tracing of $\mathrm{SN}$ fiber from bone to DRG. The retrograde neural tracer, fluoro-ruby, was injected into the medullary cavity of tibiae. Two days after tracer injection, DRGs (L3-5) were harvested and subjected to immunohistochemical analyses. Sections of DRG were immunostained with anti-CGRP and anti-TRPV1 antibody and examined under a confocal laser scanning microscope (x 400). Arrow: Fluoro-ruby ${ }^{+}, \mathrm{TRPV}^{+}$, and $\mathrm{CGRP}^{+} \mathrm{SN}$ fiber.

\section{Figure 2}

A. Radiograph and histology (x 40, HE) of tibiae injected with LLC cells at day 7 and 14. Arrows: osteolytic lesions. Asterisks: LLC tumor.

B. Increase in numbers of TRAP ${ }^{+}$osteoclasts in bone injected with LLC cells over time.

* $\mathrm{p}<0.05$ vs day 0 (mean $\pm \mathrm{SE}, \mathrm{n}=5)$.

C. Increase in numbers of $\mathrm{CGRP}^{+}$and $\mathrm{TRPV} 1^{+} \mathrm{SN}$ fibers in bone injected with LLC cells. Mice were sacrificed at day 14 and sections of bone were immunostained with anti-CGRP 
and anti-TRPV1 antibody. * $p<0.01$ vs sham (mean \pm SE, $n=5$ ).

D. Thermal hyperalgesia in mice intratibially injected with PBS (sham) or LLC cells (tumor). Assay was conducted in a blind manner, * $p<0.05$ vs sham (mean $\pm S E, n=10$ ).

E. Frequency of flinching in mice injected with PBS (sham) or LLC cells (tumor) intratibially during 10 min observation. * $\mathrm{p}<0.05$ vs sham (mean $\pm S E, n=10$ ).

F. Duration of flinching in mice injected with PBS (sham) or LLC cells (tumor) intratibially during 10 min observation. ${ }^{*} \mathrm{p}<0.05$ vs day sham (mean $\pm S E, n=10$ ).

\section{Figure 3}

A. Acidic microenvironment of LLC tumor in bone. Mice were injected with DAMP before sacrifice at day 14 . Sections of bone were incubated with rabbit anti-dinitrophenol antibody and counterstained with methyl green (x 200). Brown indicates acidic area.

B. Acidic microenvironments associated with $\mathrm{TRAP}^{+}$osteoclasts (x 200).

C. Effects of bafilomycin A1 on thermal hyperalgesia in mice injected with PBS (sham) or LLC cells (tumor) intratibially. Bafilomycin A1 (25 $\mu \mathrm{g} / \mathrm{kg} / \mathrm{day}, \mathrm{sc})$ was given to mice once a day from day 7 to day 14 . ${ }^{*} p<0.05$ vs control sham group (mean $\pm S E, n=8$ ). ${ }^{*} p<0.05$ vs control tumor group (mean $\pm \mathrm{SE}, \mathrm{n}=8$ ).

D. Effects of bafilomycin A1 on the frequency of flinching in mice injected with PBS (sham) or LLC cells (tumor) intratibially. Flinching test was conducted at day 14 . ${ }^{*} \mathrm{p}<0.05$ vs control (mean \pm SE, $n=10)$.

\section{Figure 4}

A. Thermal hyperalgesia in WT mice intratibially injected with PBS (sham) or LLC cells (tumor). ${ }^{*} \mathrm{p}<0.05$ vs sham (mean $\pm \mathrm{SE}, \mathrm{n}=10$ ).

B. Thermal hyperalgesia in TRPV1-/- mice intratibially injected with PBS (sham) or LLC cells (Tumor). 
C. Frequency of flinching in WT and TRPV1-/- mice intratibially injected with PBS (sham) or LLC cells (tumor) during 10min observation at day 14. ${ }^{*} \mathrm{p}<0.05$ vs WT (mean $\pm \mathrm{SE}, \mathrm{n}=10$ ).

D. Duration of flinching in WT and TRPV1-/- mice intratibially injected with PBS (sham) or LLC cells (tumor) during 10min observation at day $14 .{ }^{*} \mathrm{p}<0.01$ vs WT (mean $\left.\pm \mathrm{SE}, \mathrm{n}=10\right)$.

\section{Figure 5}

A. IHC of $\mathrm{pERK}^{+}$neurons (arrow) in DRG in WT mice intratibially injected with PBS. DRGs (L3-L5) ipsilateral to LLC cell-injected tibiae were harvested at sacrifice at day 14. Sections were stained with anti-pERK antibody (1:1,000) overnight at RT (x 100).

B. IHC of pERK ${ }^{+}$neurons (arrows) in DRG in WT mice intratibially injected with LLC cells.

C. IHC of pERK ${ }^{+}$neurons (arrow) in DRG in TRPV1-/- mice intratibially injected with PBS.

D. IHC of pERK ${ }^{+}$neurons (arrow) in DRG in TRPV1-/- mice intratibially injected with LLC cells.

E. Numbers of $\mathrm{pERK}{ }^{+}$neurons/CGRP ${ }^{+} \mathrm{SNs}$ in $\mathrm{DRG}$. ${ }^{*} \mathrm{p}<0.05$ vs sham (mean $\pm \mathrm{SE}, \mathrm{n}=10$ ), ${ }^{\#} p<0.05$ vs WT tumor group (mean $\pm \mathrm{SE}, \mathrm{n}=8$ ).

\section{Figure 6}

A. IHC of c-fos neurons (arrow) in spinal dorsal horn in WT mice intratibially injected with PBS. Spinal dorsal horns ipsilateral to LLC cell-injected tibiae were harvested at sacrifice at day 14 . Sections were stained with anti-c-fos antibody $(1: 2,000)$ overnight at RT $(x$ 100).

B. IHC of c-fos neurons (arrows) in spinal dorsal horn in WT mice intratibially injected with LLC cells.

C. IHC of c-fos neurons (arrow) in spinal dorsal horn in TRPV1-/- mice intratibially injected with PBS.

D. IHC of c-fos neurons (arrow) in spinal dorsal horn in TRPV1-/- mice intratibially injected with LLC cells.

E. Numbers of $c$-fos neurons $/ \mathrm{CGRP}^{+} \mathrm{SNs}$ in spinal dorsal horn. ${ }^{*} \mathrm{p}<0.05$ vs sham (mean $\pm \mathrm{SE}$, 
$n=10),{ }^{\#} p<0.05$ vs WT tumor group (mean $\pm S E, n=8$ ). 
A
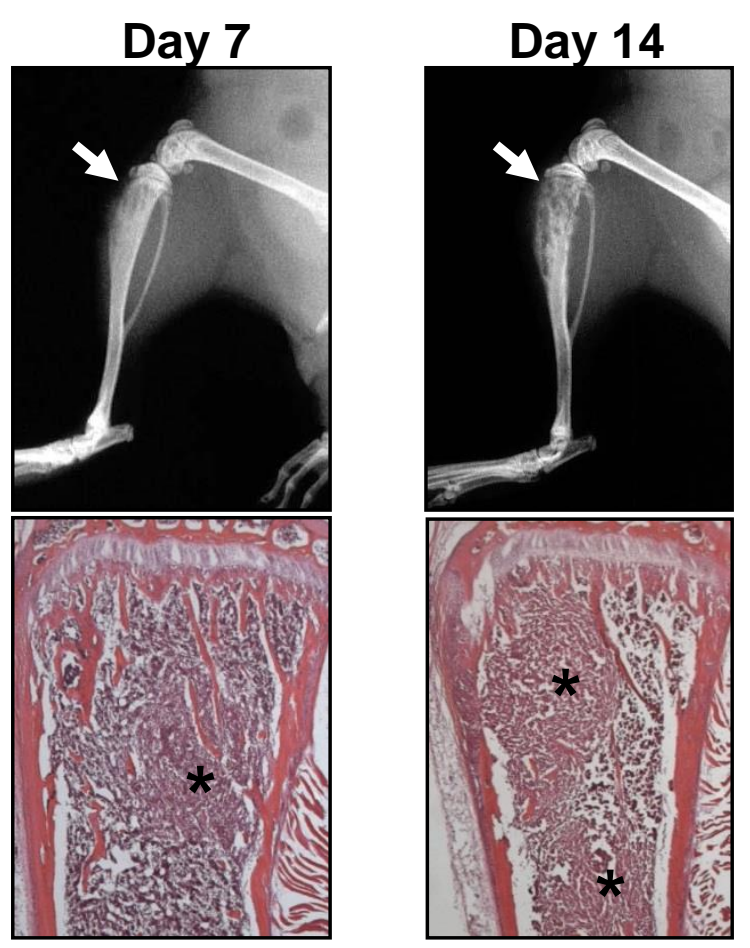

No CGRP ${ }^{+}$, TRPV1$^{+}$fibers

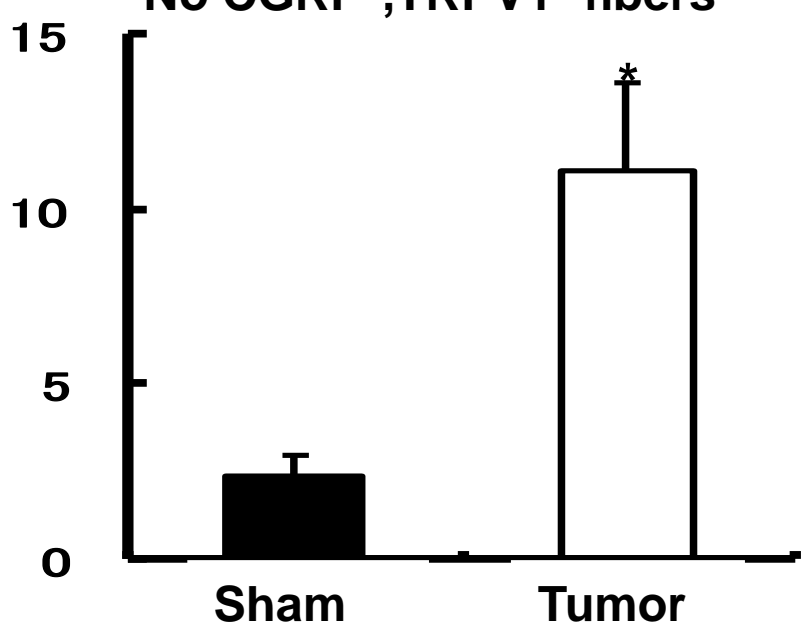

$E$
20
15
10
5
0

Frequency/10min

1

Tumor 口Sham

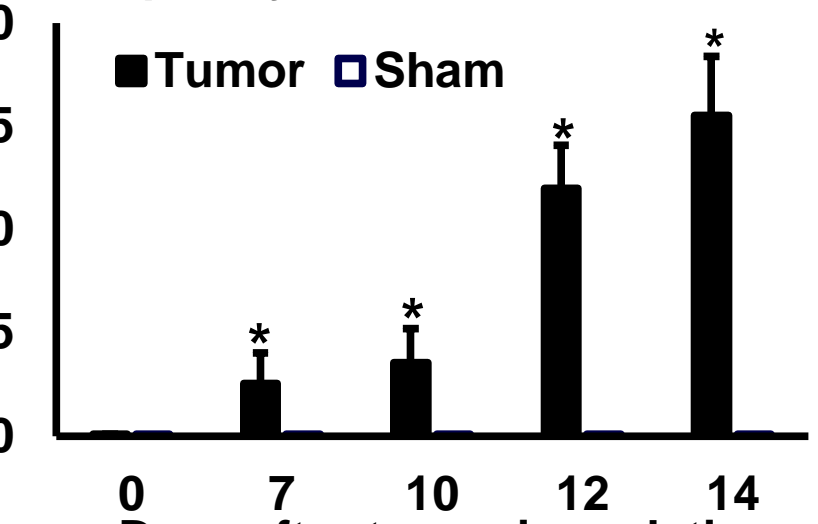

Days after tumor inoculation

Figure 2

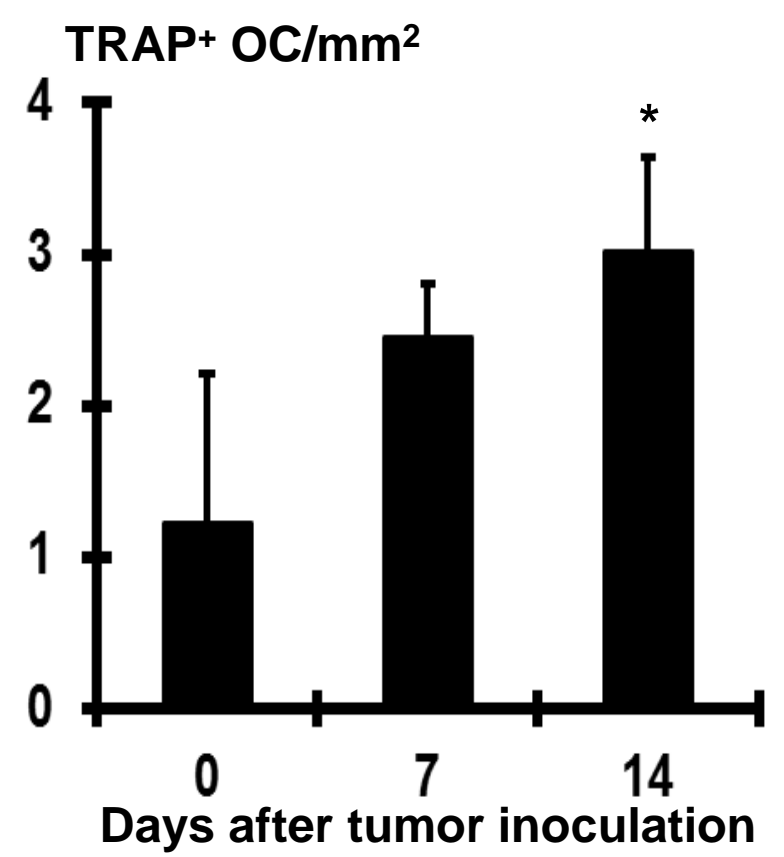

D

Time required for paw withdrawal (s)
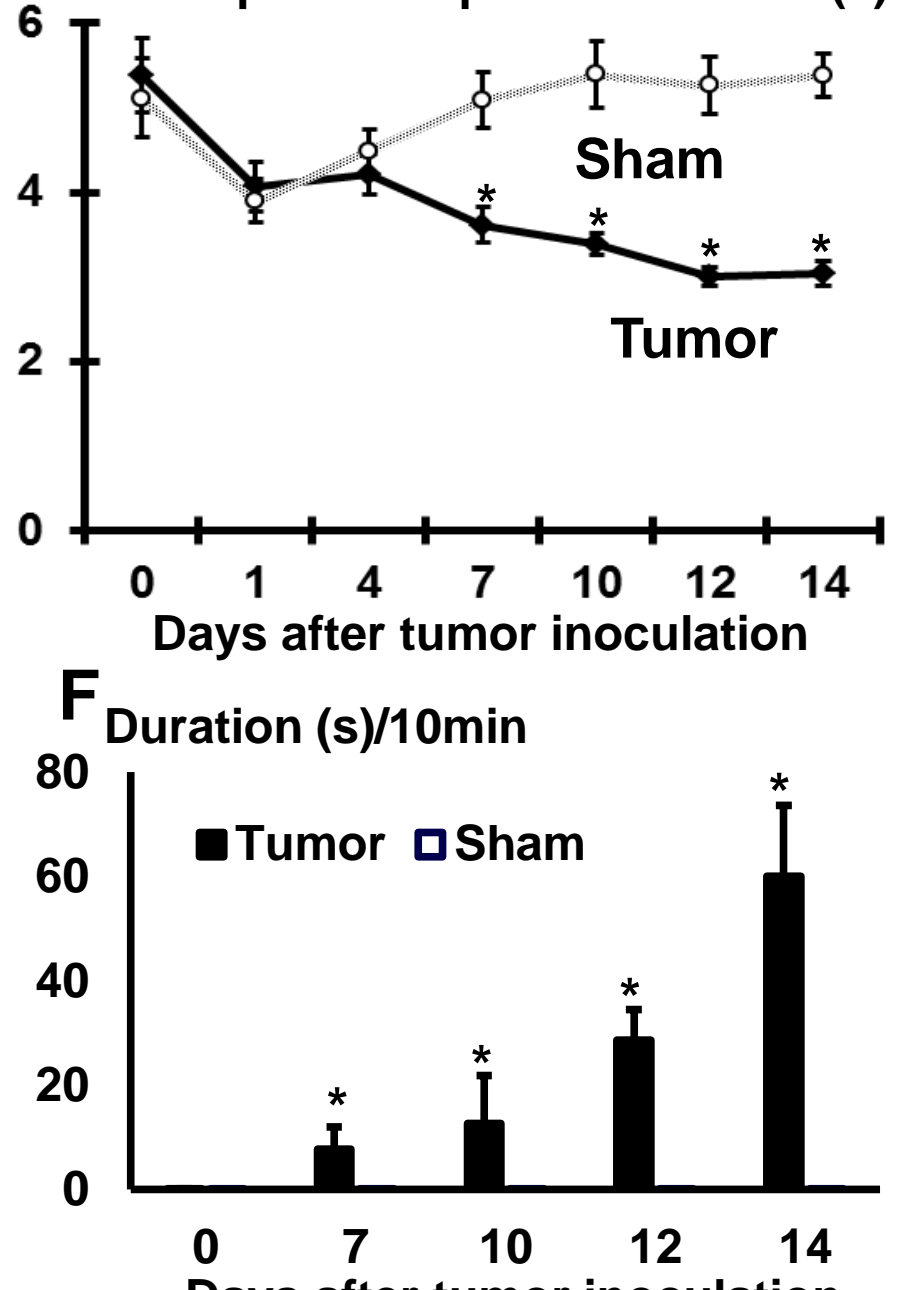

Days after tumor inoculation 

A
WT
B
TRPV1-I-

Time required for paw withdrawal(s)

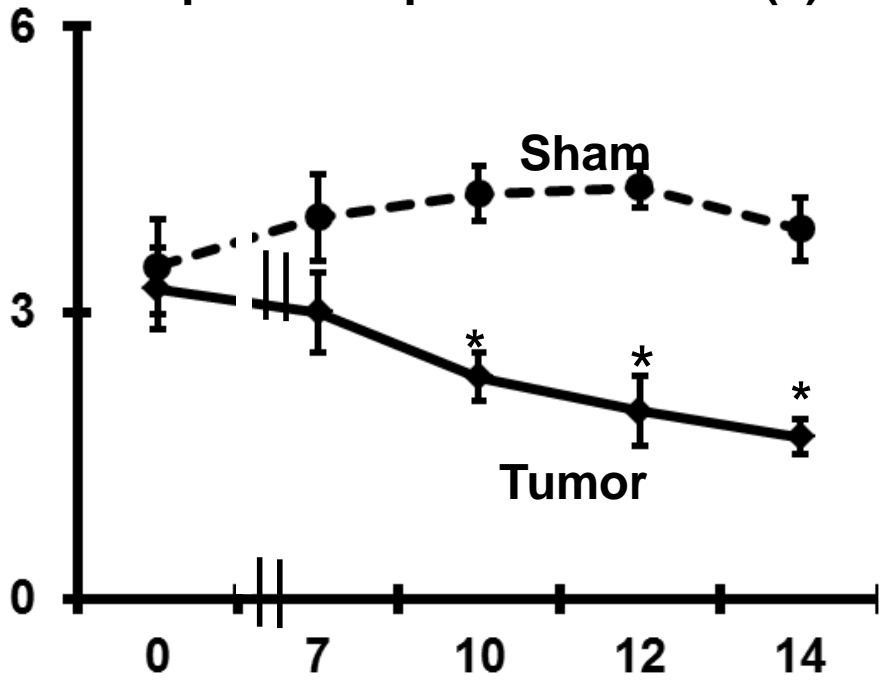

Days after Tumor Inoculation
Time required for paw withdrawal(s)

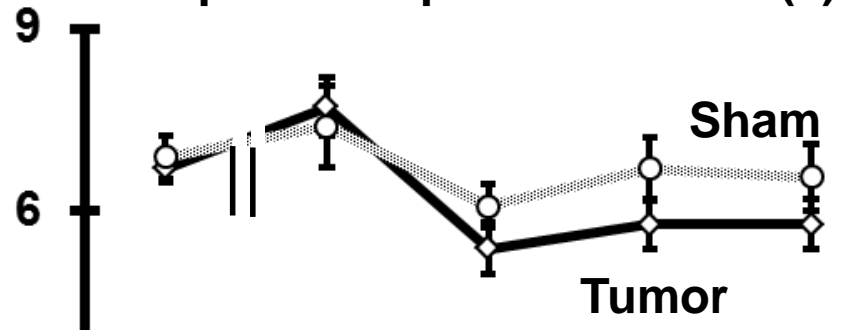

0

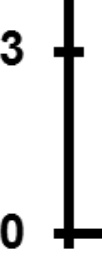

0

Days after Tumor Inoculation

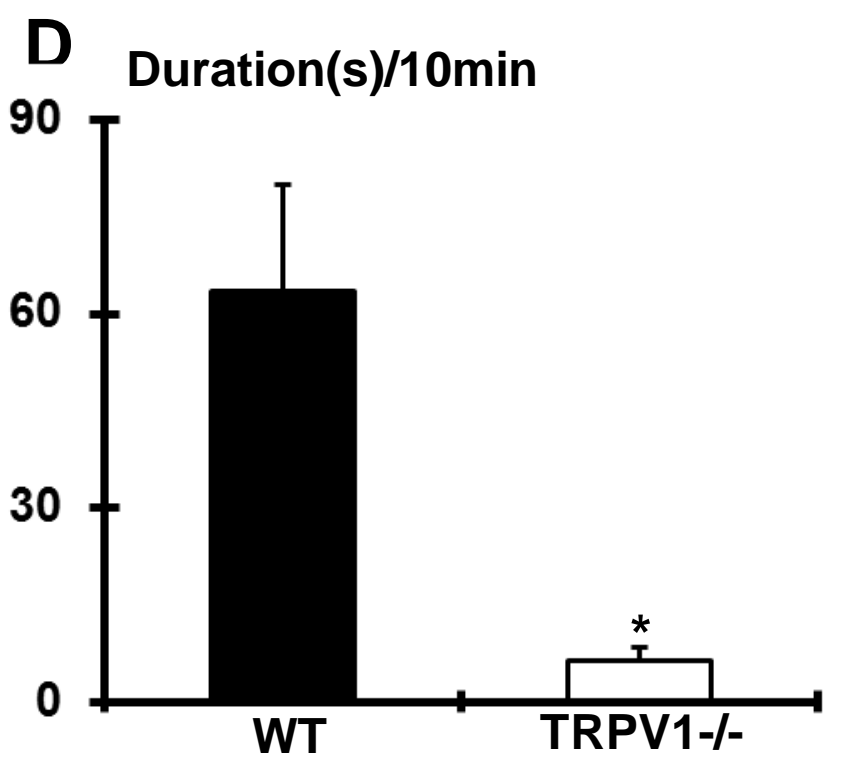

Figure 4 

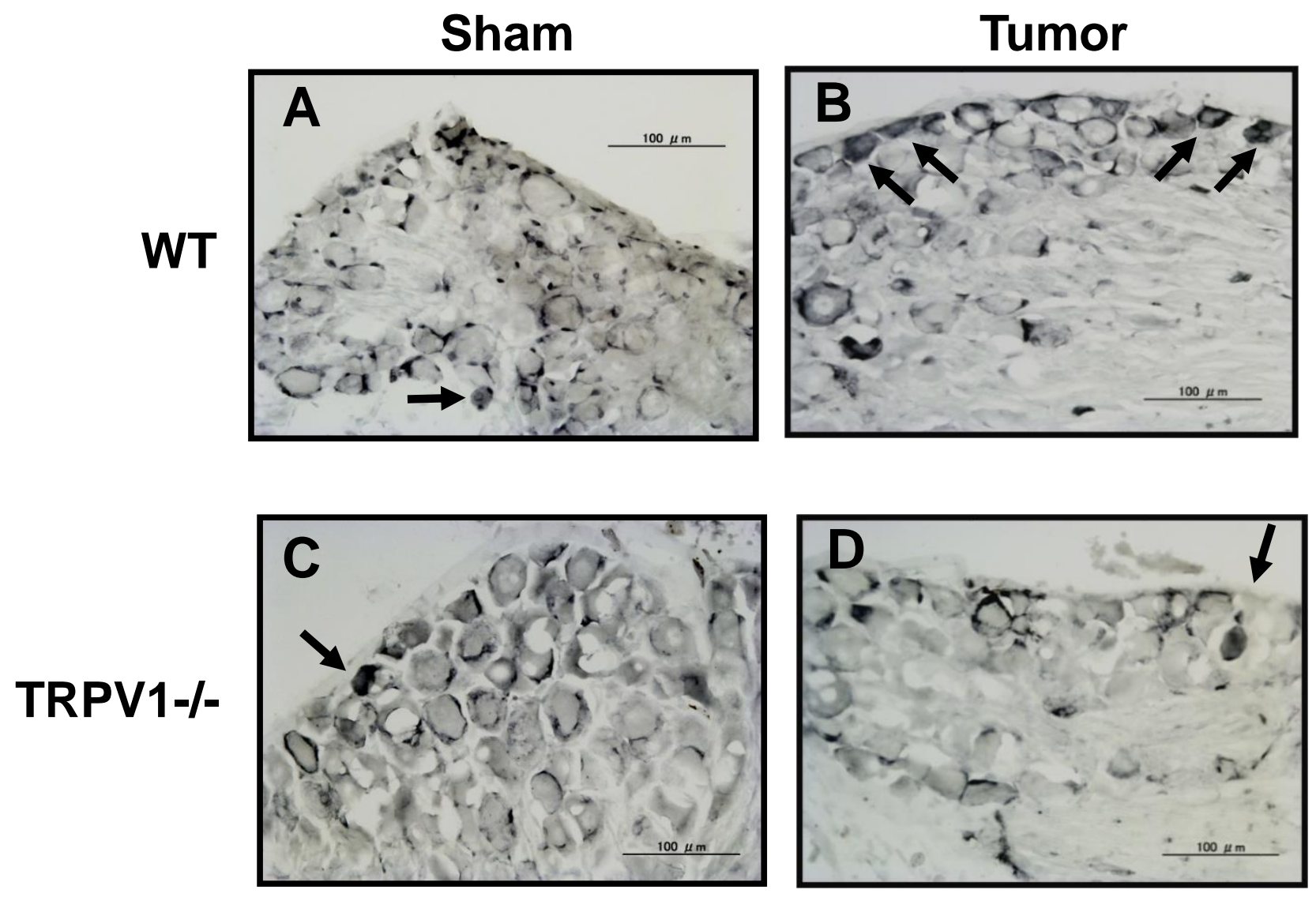

E

No. pERK ${ }^{+}$neurons/CGRP ${ }^{+}$SNs (\%)

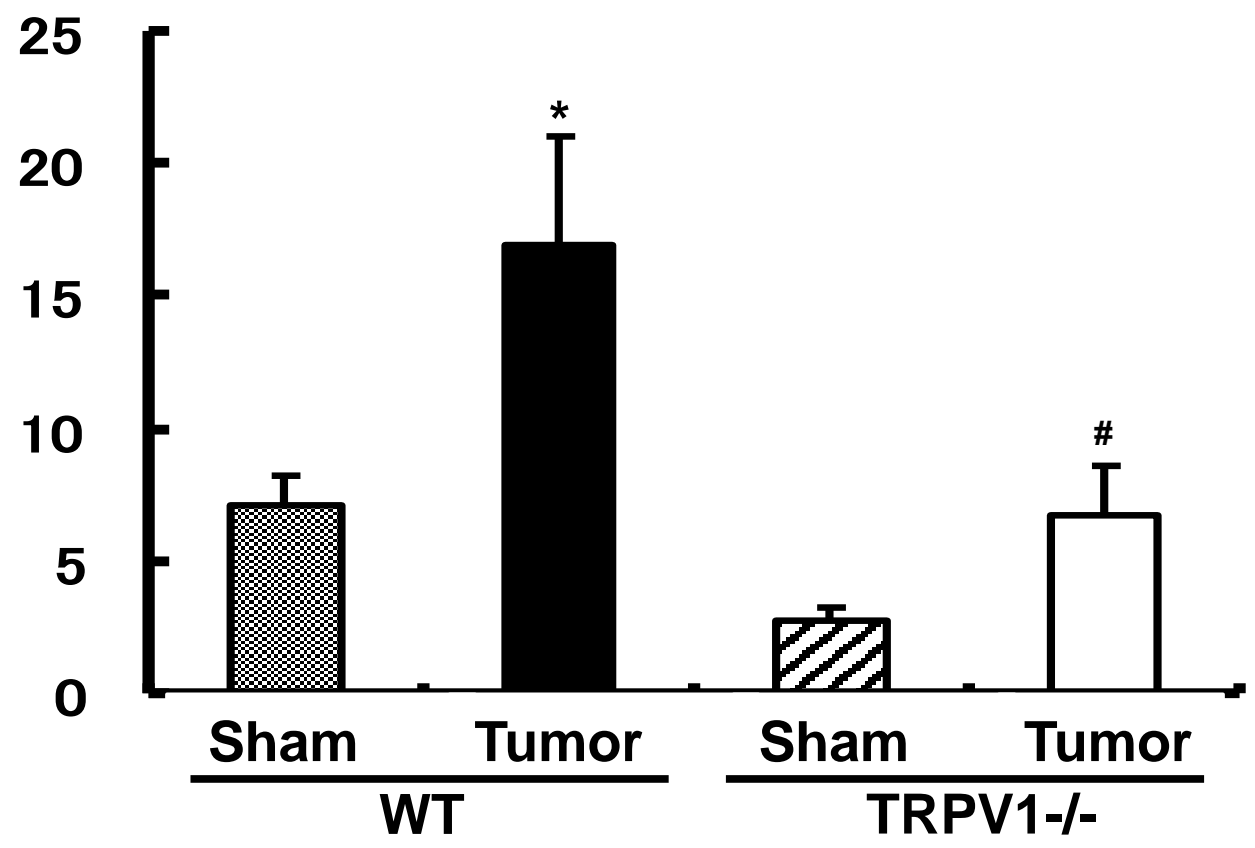

Figure 5 

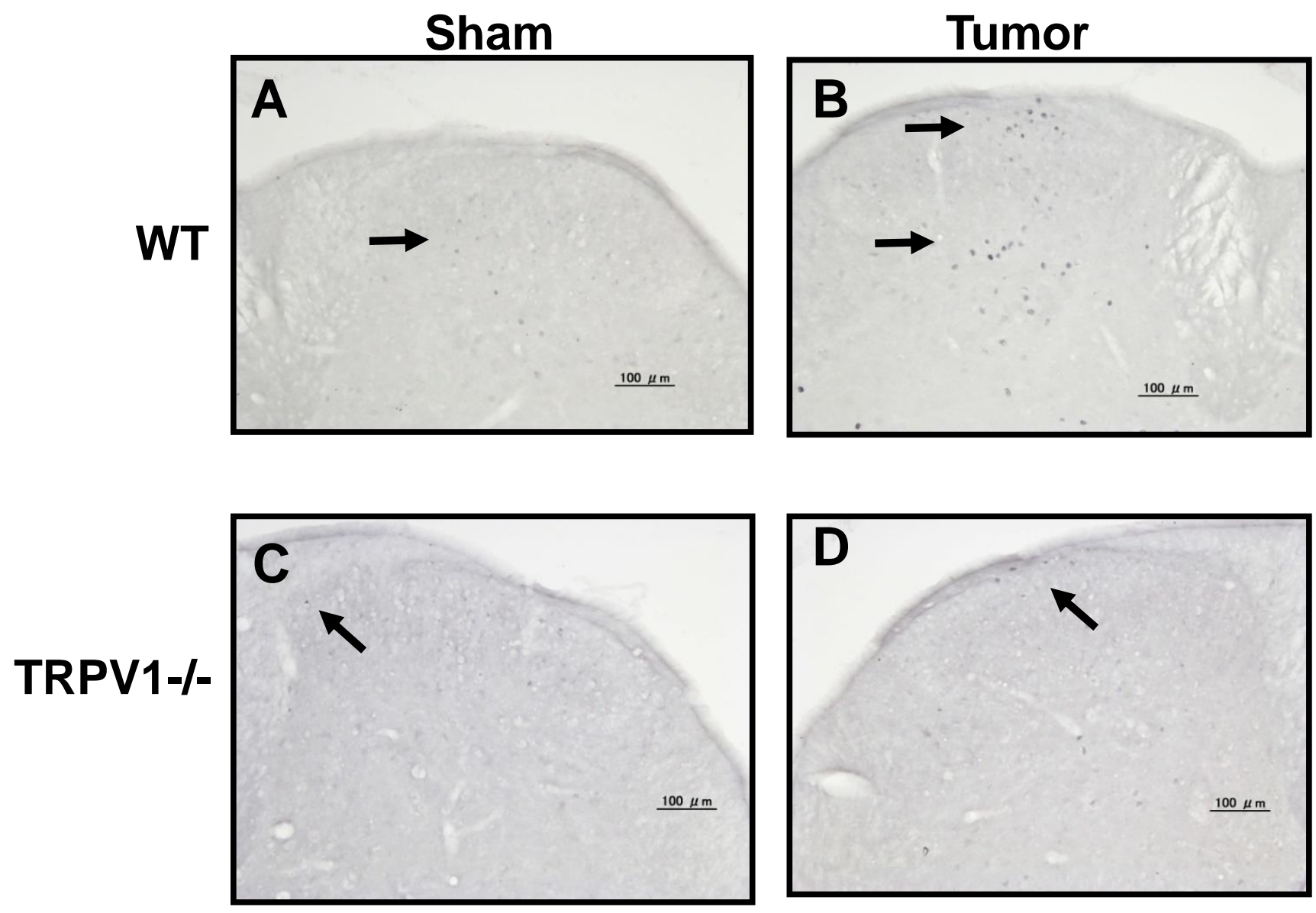

E

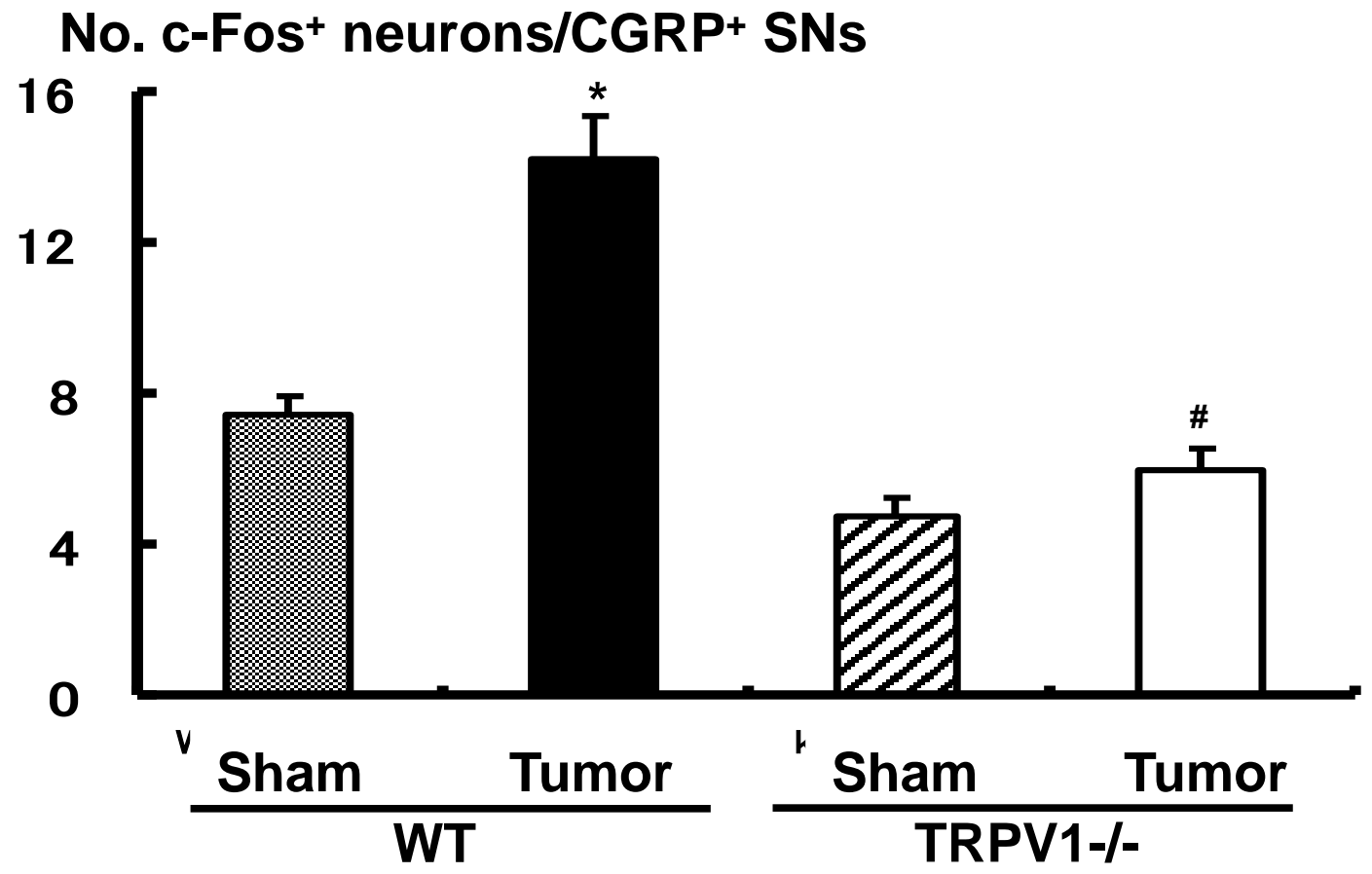

Figure 6 